\title{
HYDATID LIVER DISEASE - A REVIEW OF THE LITERATURE
}

\author{
Valentin Ignatov', Nikola Kolev', Georgi Ivanov', Anton Tonev', Vilian Platikanov², \\ Aleksandar Zlatarov ${ }^{1}$, Georgi Todorov ${ }^{1}$, Krasimir Ivanov ${ }^{1}$ \\ ${ }^{1}$ First Clinic of Surgery and ${ }^{2}$ Department of Anesthesiology and Intensive Care, \\ St. Marina University Hospital of Varna
}

\begin{abstract}
Hydatid cyst disease of the liver is a worldwide parasitosis with significant debilitating effect on the patients. In Bulgaria, the most important biological reservoir and the final host is the dog. The distribution of the disease in the territory of the country is variable. Advances of laboratory and imaging diagnostic methods provide new options for multimodal approach. Surgery has been mainstay of treatment, however, new minimally invasive methods such as laparoscopic surgery and PAIR present with advantages in selected patients. Perioperative therapy diminishes postoperative relapses. In the present review, new data about the epidemiology and recent achievements in diagnosis and management of hydatid liver disease are presented.
\end{abstract}

Key words: hydatid liver disease, epidemiology, diagnosis, treatment, Bulgaria

\section{INTRODUCTION}

Hydatid cyst disease of the liver is a cosmopolitan parasitosis (13). Echinococcus granulosus (E. granulosus) is the species that prevails in most countries around the world and, especially, in the Mediterranean region (7). It is one of the most severe infestations in humans. Compared to other zoonosis, hydatic cyst disease caused by $E$. granulosus most often leads to prolonged disability, frequent recurrences and is associated with high mortality rates. All this makes it a serious medicosocial and economic problem for Bulgaria. Data from the literature available suggest that liver is the most commonly affected organ (2).

Address for correspondence:

Georgi Ivanov, MD, PhD

First Clinic of Surgery,

St. Marina University Hospital of Varna

1 Hristo Smirnenski Str., 9010 Varna, Bulgaria

e-mail:ghivanov@abv.bg

Received: October 01, 2012

Accepted: March 04, 2013
The leading treatment protocol for this disease is mainly surgical and/or mini-invasive combined with adjuvant systemic therapy. This disease is a subject of surgical treatment in all the surgical clinics in Bulgaria. It can affect people of any age groups and thus be particularly debilitating for children. Nowadays there are, however, no established protocols for diagnostic and surgical behaviour.

\section{Historical data}

The disease is known since antiquity onwards. Hippocrates, Aristotle and many others considered echinoccocic blisters to be enlarged and extended lymph nodes. In 1681, Redi discovered that these cysts are actually live parasites (6). Subsequently, articles on this parasitosis of other authors have been increasingly published in the specialized literature.

\section{Taxonomy}

The nomination of genus Echinococcus was first introduced in 1801 (21).

E. granulosus is the most widespread species. So far, 10 strains are included here listed from G1 to G10 (21). These different genetic E. granulossus strains include two sheep strains (G1 and G2), two 
bovine (G3 and G5), one horse strain (G4), one camel (G6), two pig (G7 and G9) and two deer strains (G8 and G10). Up-to-date, only a few strains are studied in details (14). Therefore, additional studies need to be conducted in order to obtain more detailed information about the hosts, their geographic distribution and genetic characteristics depending on their endemic regions.

A survey including 9,970 patients (from regions in South America, Africa, Europe and Australia, where the sheep strain is widespread and infection is endemic) shows that liver is affected twice more often than lungs (13). The situation is different in patients who are infected but remain asymptomatic (24). Ultrasound and x-ray examinations of almost 10,000 individuals living in endemic regions of Argentina and Uruguay demonstrate a more frequent liver involvement by 6 and 12 times, respectively, when compared to pulmonary affection (13). The explanation of the higher incidence rate of hepatic damage in asymptomatic patients compared to hospitalized patients is that hydatid cysts located in the lungs cause a higher morbidity rate than these located in the liver $(14,25)$.

\section{Epidemiology and parasitology}

In Bulgaria, final hosts are considered the source of infection as dog is the most common. Epidemiological surveys show that the rate of infestation is highest amongst shepherd dogs $(16,94 \%)$, stray dogs $(12,12 \%)$, domestic dogs $(8,16 \%)$, and hunting dogs $(5,66 \%)$. However, stray dogs are considered the most risky.

\section{Morbidity rates in Bulgaria}

Data analysis on hydatid cyst disease in humans for a 52-year period (1954-2006) shows a gradual decrease of morbidity rate after the start of organized control measures (26). Morbidity rates decrease from $6,5 \%_{\text {ооо }}$ to $1,61 \%_{\text {оо }}$ for the period between 1954 and 1980. A drastic morbidity rate increase in humans is registered after 1992, exceeding the initial value of $6,5 \%{ }_{\text {ооо }}$. During 1998 , the morbidity rate increases up to $8,47 \%_{\text {ооо }}$ (26). The territorial distribution of the disease in Bulgaria is uneven in different regions of the country. Highest morbidity rates are registered in Bourgas region, an average for the period of $12,29 \%_{\text {ооо }}\left(27,7 \%_{\text {ооо }}\right.$ in 1998); in Sliven region - $11,81 \%$ ooo; in Plovdiv region - $8,22 \%_{\text {oоo }}$, and in Stara Zagora region - $8,05 \%_{\text {oоo }}$. Lowest morbidity rates are registered in Vidin region - $0,75 \%$; in Sofia city - $1,95 \%_{{ }_{\text {ooo }}}$, and in Montana region $1,78 \%_{\text {ооо }}$ (4). The most affected population is aged between 40-49 years and 50-59 years. Unfortunately, hydatid cyst disease in the last 10 years affects 1092 children aged 10-19 years, which accounts for $21,68 \%$ of all the operated patients (4). The earliest age is 1,5 years. During the last 10 years, there is a reversed tendency in accordance to urban and rural patients' distribution (urban - 54,51\% and rural - 45,49\%) (19). There are more females than males $(54,73 \%$ versus $45,23 \%)(4,19)$. Patients' distribution according to the professional occupation demonstrates a significant number of people related to agricultural work. The analysis of education degree demonstrates that most patients $(53,60 \%)$ have elementary only or no education at all, 40,96\% have secondary education and only $6,44 \%$ have higher education (4). The dog is the main definitive host and biologic reservoir of invasion. Most affected are shepherd (up to 78\%), homeless (57\%), domestic (31\%) and hunting dogs (16\%) (24). Of the intermediate hosts, sheep is the most important factor for dissemination of the disease. Fertility echinococci occur most often in sheep and pig. Cattle play a smaller role, since $90 \%$ of them in developing cysts are sterile. Processed data from slaughter meat of different species for the last six years display an increasing contamination of cattle - from $9,17 \%$ to $17,91 \%$, of sheep - from $5,17 \%$ to $7,5 \%$ and of pig - from $0,8 \%$ to $2,19 \%$. Echinococcosis in cattle is registered in the country, however, in some regions it reaches up to $37 \%$ (in Sofia), 31,6\% (in Bourgas), 28\% (in Haskovo) while that in sheep - up to $29 \%$ (in Vratsa), 24\% (in Pernik), 23\% (in Sliven), and $32 \%$ (in Varna) (4).

The incidence rate of echinococcosis among the population of different countries varies widely. In 2003, 374 patients infected with echinococcosis were registered in the European Union and Norway. Data about the incidence rate of hydatid disease in European countries show significantly lower values. Sporadic, mostly imported cases occur in Belgium, Luxembourg, the Netherlands, and Sweden. Low incidence rate below $1,0 \%{ }_{000}$ is noted in Austria, Switzerland, Germany, and Great Britain. Countries with high morbidity rate are Spain $\left(1,8 \%{ }_{000}\right.$ in 1986-1990), in the affected areas of Italy $\left(2.5 \%{ }_{000}\right.$ in $\left.1980-1984\right)$, 
Valentin Ignatov, Nikola Kolev, Georgi Ivanov et al.

Romania (1,6-6,4\% ${ }_{\text {ooo }}$ in 1992), in different areas of Turkey $\left(0,87-20,0 \%{ }_{000}\right.$ in 1993), in different areas of Greece $\left(11,7-13,3 \%_{\text {oоo }}\right.$ in 1981-1983 and reduced down to $3,67 \%{ }_{000}$ in 1993 after implementing the organized campaign against echinococcosis). A comparison of official data in Bulgaria with those in other European countries demonstrates a very unfavourable situation of echinococcosis in our country. In 1976, Bulgaria comes second in the world after Iceland with morbidity rate of $1,24 \%{ }_{000}$ (of which, 140 operated patients) $(4,18)$.

Echinococcosis has a chronic course. It can be asymptomatic for years (8). The symptoms result from mechanical trauma and toxico-allergic effects. According to different authors, clinical hepatic hydatid disease occurs in two to four stages. Three-stage classification of disease is the most accurate and useful for practice.

1. First stage (latent) - from infestation to onset of symptoms. A study in Italy reveals that $60 \%$ of the patients diagnosed with hepatic hydatid disease are asymptomatic (24). Ultrasound studies in South America show that the average diameter of echinoccocic cysts in asymptomatic carriers is significantly smaller (about $4 \mathrm{~cm}$ ) than that in symptomatic patients (about 10 $\mathrm{cm})$ (13).

2. Second stage - manifestation of clinical symptoms. Clinical symptoms appear after highly variable incubation period of several months to several years. Out of 28 studied patients with hepatic echinococcosis in Argentina, 21 (75\%) remain asymptomatic during the observation period for 10-12 years after initial diagnosis, while 7 (25\%) of them develop symptoms related to the localization of the cysts (13). Leading symptoms at diagnosis are: jaundice, abdominal pain, gastrointestinal and upper abdominal discomfort (nausea, vomiting, bloating, anorexia), acute pancreatitis and portal hypertension.

3. Third stage - occurrence of complications. Suppuration is one of the most common complications of liver echinococcosis $(15,17)$. A higher frequency of ruptures compared with suppurations of echinoccocus cysts (33,1\% versus $29,6 \%$, respectively) is reported (17). In case of ruptured suppurated cyst in the biliary system there is a significant deterioration of prognosis and treatment outcomes (18).

\section{Classification}

Several classifications of hepatic hydatid disease are proposed. Here belong Gharbi and WHO-IWGE classifications (3).

\section{DIAGNOSIS}

\section{Laboratory methods}

Standard diagnostic approach to hepatic hydatid disease involves a combination of imaging techniques as well as laboratory and serological analysis (14).

\section{Laboratory methods include a set of:}

i) biochemical examinations: haematological parameters - eosinophilia (15-50\%), accelerated ESR, and leukocytosis; syndrome of 'small' liver failure (bilirubin, AST, ALT, alkaline phosphatase, GGTP, and proteins), and

ii) immunological examinations. The biological diagnosis is based on serological tests, ELISA, immunofluorescence reaction, passive haemagglutination reaction, latex agglutination reaction and immunoelectrophoresis reaction in double diffusion gel. Complement fixation is of poor sensitivity and specificity. Indirecthaemagglutination reaction possesses a good sensitivity, but there is a large percentage of false-positive results. According to different authors, immunoelectrophoresis and ELISA show the best results with a sensitivity of $90 \%$ for liver localizations of hydatid cysts $(5,7,14,22)$.

\section{Imaging methods}

Conventional and intraoperative abdominal ultrasound is useful for determining the internal structure, size, number and location of cysts, presence of complications with sensitivity of $90-98 \%$ according to literature data. The method is suited for planning the appropriate treatment and evaluation of the therapy.

Computed tomography (CT) provides information equivalent to that of a conventional or intraoperative ultrasonography. However, it shows a more detailed localization and depth of the hydatid cyst. CT is the method of choice when a suppurated hydatid cyst is suspected (6). CT has accuracy of $100 \%$ in the diagnosis of hydatid cyst disease (2). CT 
is superior to ultrasound in the visualization of gas cysts, calcifications, and more precisely determines the position of hydatid cysts (24). Numerous studies confirm the higher sensitivity of CT than that of ultrasound in the diagnosis of hydatid cyst $(1,7)$. CT is a reliable method to detect small cysts compared with ultrasound (24).

MRI provides good structural details about the hydatid cyst, however, its use is not economically viable. Some authors fail to establish any significant superiority of the method in the diagnosis of hepatic hydatid cyst and, therefore, it does not apply everywhere. The main advantage of MRI over CT and ultrasound is in severe cases of complicated hydatid disease with suspected rupture of the cyst into the biliary tree (12).

\section{TREATMENT}

Under the new standards for clinical approach to hepatic hydatid disease, there is no single 'golden rule' as it is important that treatment be individualized for each patient and each cyst (1,2,7,14,19-25).

\section{Conventional surgery}

Operational methods are conservative (processing and closing the cavity with or without resection of portions of the capsule) and radical (surgery of liver tissue). The principles of surgical approach to hepatic hydatid disease include neutralization of the parasite, evacuation of the cyst, germ capsule removal and treatment of the residual cavity. Total surgical removal that is the ideal treatment option can be achieved through elimination of germinal layer, daughter cysts and fluid, or by resection of the intact cyst including the pericystic area. Surgery is indicated for the removal of large cysts with multiple daughter cysts, single and superficially located liver cysts, which may rupture spontaneously or because of trauma, suppurated cysts, cysts in the biliary tract communication causing the compression of adjacent organs. Contraindications of surgical treatment include lack of informed consent by the patient, older age, pregnancy, comorbidity (cardiovascular diseases, liver disease, diabetes mellitus), multiple hydatid disease, difficultly accessible cysts, and non-viable cysts in patients with very small cysts (2).
Major postoperative complications reported by different authors are mainly inflammatory processes $(6,15,20,27)$. There are liver abscesses in $2 \%$, wound infections in $5 \%$, and pneumonia in $2-4 \%$ of the patients having undergone open surgery.

\section{Laparoscopic surgery}

Laparoscopic procedures in the treatment of liver echinococcus include complete excision in the cyst, unrufing, evacuation and obliteration in cyst cavity $(10,15,16,26)$. Laparoscopic approach is a miniinvasive method with short postoperative hospital stay and minimal risk of wound complications $(10,11)$. Cyst localization and presence of complications create technical difficulties in performing the manipulation. However, many authors recommend this method. Contraindications of laparoscopic surgery are acute cardiopulmonary failure with $\mathrm{CO}_{2}^{-}$ induced pneumoperitoneum, previous abdominal surgery causing adhesions and reducing inspection, recurrent cysts, cysts located $1 \mathrm{~cm}$ in depth and hidden risk of bleeding $(10,11)$.

Abdominal dissemination and biliary leakage are severe complications of the laparoscopic procedure. In non-selected patients, poor imaging and false method increase conversion rate. According to literature data available, postoperative relapse occurs in $2-3 \%$ of the patients until the sixth month after the intervention $(2,27)$.

\section{PAIR}

Percutaneous approach under ultrasound guidance plays an important role in the treatment of liver hydatid disease during the last two decades $(1,16,25)$. This method comprises 4 steps: $1^{\text {st }}$ - punction in cyst, $2^{\text {nd }}$ - aspiration of cyst fluid, $3^{\text {rd }}$ - injection of scolics agent, and $4^{\text {th }}$ - reaspiration. The scolics fluid is a hypertonic sodium chloride solution (20\% solution). The efficacy of the method is confirmed by many studies. Percutaneous drainage is an effective and safe method with a low rate of complications. This method is used in inoperable patients and in patients who refuse surgery.

PAIR is used in the treatment of cysts sized over $5 \mathrm{~cm}$, of cyst type I, type II, and type III of Gharbi except a 'honeycomb', several cysts with a diameter of $5 \mathrm{~cm}$ situated on different liver segments. PAIR can be used in patients planned for surgery 
Valentin Ignatov, Nikola Kolev, Georgi Ivanov et al.

and in those who do not respond to drug therapy. Contraindications are inaccessible superficial cysts, septated cysts, cysts with echogenic lesions, calcified cysts, cysts in communication with the biliary system, and cysts with dense contents. Major complication after PAIR are the anaphylactic shock, secondary hydatid disease due to leakage of cystic fluid in the abdominal cavity and chemical cholangitis caused by contact with or penetration of scolics solution in the biliary tree.

\section{Drug therapy}

Medical treatment is based on the application of benzomidazol carbamite, albendazole and mebendazole. This therapeutic method is applied in inoperable patients and as a supportive treatment in pre- and postoperative time $(8,13,14)$. Most cysts in patients on drug therapy show degenerative changes such as reduction of volume and/or morphological alterations such as consolidation, membrane separation and cyst calcification. Some authors report a parasitostatic effect in $61-68 \%$, predominantly in smaller and younger cysts $(1,24)$. Indications for use of drug therapy are multiple and relapsed echinococcosis, prevention of secondary echinococcosis, presence of contraindications or refusal of surgery $(2,4,7,24)$. Contraindications are purulent cysts, cysts with rupture into the biliary tree, giant cysts, devitalized or calcified cysts, septated cysts, chronic liver disease, anemia, pregnancy, and lactation.

\section{CONCLUSION}

The standard diagnostic approach to hydatid liver disease includes a constellation of laboratory and imaging methods. ELISA provides the best diagnostic results. Ultrasound is of high sensitivity while CT is more sensitive for small cysts and plays an important role in planning the radical surgery. Surgery is the golden standard for treatment of liver hydatid cyst disease. Perioperative therapy is advisable and helps at reducing the number of recurrences. The minimally invasive methods such as laparoscopic surgery and PAIR are safe and effective alternatives.

\section{REFERENCES}

1. Agaba, E. A., D. R. Camacho, P. Vemulapalli. Hydatid cyst disease revisited: optimal management of complex liver disease.- South. Med. J., 104, 2011, No 4, 254-255.

2. Agayev, R. M. Diagnosis and surgical treatment of complicated liver echinococcosis.- Turk. J. Gastroenterol., 13, 2002, No 1, 11-16.

3. Alempijević, T., N. Kovacević, A. SokićMilutinović, A. Pavlović-Marković, M. Milicević. Hepatic echinococcal cyst - personal experience and report of a new classification system according to WHO.- Acta Chir. Iugosl., 58, 2011, No 3, 37-41 (in Serbian).

4. Boeva-Bangezova, V. G. The achievements and problems in controlling human echinococcosis in Bulgaria.- Med. parazitol. (Mosk.), 1991, No 1, 4345 (in Russian).

5. Chouillard, E., A. Gumbs, P. Fangio, A. Torcivia, C. Tayar, A. Laurent, et al. Liver resection for cystic lesions: a 15-year experience.- Minerva Chir., 65 , 2010, No 5, 495-506.

6. Cubas-Castillo, R., C. O. Brain, R. López-Guillemain, E. A. Ballarino. Hydatid liver disease: our 25year experience.- Cir. Cir., 79, 2011, No 4, 306-312.

7. Dakkak, A. Echinococcosis/hydatidosis: a severe threat in Mediterranean countries.- Vet. Parasitol., 174, 2010, No 1-2, 2-11.

8. Durakbasa, C. U., G. A. Tireli, V. Sehiralti, S. Sander, A. N. Tosyali, M. Mutus. An audit on pediatric hydatid disease of uncommon localization: incidence, diagnosis, surgical approach, and outcome.J. Pediatr. Surg., 41, 2006, No 8, 1457-1463.

9. Foster, E. N., G. Hertz. Echinococcus of the liver treated with laparoscopic hepatectomy.- Perm. J., 14, 2010, No 2, 45-46.

10. Gastaca, M., A. Ventoso, J. González, J. Ortiz De Urbina. Laparoscopic surgery of hepatic hydatid cyst.- Cir. Esp., 88, 2010, No 1, p. 62; author reply p. 64 (in Spanish).

11. Goldin, S. B., J. J. Mateka, M. J. Schnaus, S. Dahal. Laparoscopic drainage of a hepatic echinococcal cyst: a case report.- Case Rep. Gastrointest. Med., 2011: 107087. doi: 10.1155/2011/107087.

12. Heirwegh, G., B. Claikens. Type I hydatid cyst of the liver: typical MRI features.- JBR-BTR, 88, 2005, No 3, 136-137.

13. Larrieu, E., F. Zanini. Critical analysis of cystic echinococcosis control programs and praziquantel use in South America, 1974-2010.- Rev. Panam. Salud Publica, 31, 2012, No 1, 81-87. 
14. Mandal, S., M. D. Mandal. Human cystic echinococcosis: epidemiologic, zoonotic, clinical, diagnostic and therapeutic aspects.- Asian Pac. J. Trop. Med., 5, 2012, No 4, 253-260.

15. Mentes, A., Y. Yüzer, O. Ozbal, A. Coker, T. Ilter, A. Musoğlu. Omentoplasty versus introflexion for hydatid liver cysts.- J. Roy. Coll. Surg. Edinb., 38, 1993, No 2, 82-85.

16. Moore, J., V. Gupta, M. Y. Ahmed, B. Gociman. Hydatid cyst disease: optimal management of complex liver involvement.- South. Med. J., 104, 2011, No 3, 222-224.

17. Parlakgumus, A., A. Ezer, K. Calhshan, T. Colakoglu, S. Belli, J. Karakaya, et al. Effects of a preoperative and perioperative multidisciplinary approach for complicated liver hydatid cyst on postoperative outcomes.- Hepatogastroenterology, 57, 2010, No 104, 1493-1498.

18. Rojo-Vazquez, F. A., J. Pardo-Lledias, M. FrancosVon Hunefeld, M. Cordero-Sanchez, R. AlamoSanz, A. Hernandez-Gonzalez, et al. Cystic echinococcosis in Spain: current situation and relevance for other endemic areas in Europe.- PLoS Negl. Trop. Dis., 5, 2011, No 1, e893.

19. Romig, T., A. Dinkel, U. Mackenstedt. The present situation of echinococcosis in Europe.- Parasitol. Int., 55, 2006, Suppl. S187-S191.

20. Safioleas, M., E. Misiakos, C. Manti, D. Katsikas, G. Skalkeas. Diagnostic evaluation and surgical management of hydatid disease of the liver.- World J. Surg., 18, 1994, No 6, 859-865.
21. Sbihi, Y., D. Janssen, A. Osuna. Specific recognition of hydatid cyst antigens by serum IgG, IgE, and IgA using Western blot.- J. Clin. Lab. Anal., 11, 1997, No 3, 154-157.

22. Sbihi, Y., A. Rmiqui, M. N. Rodriguez-Cabezas, A. Orduña, A. Rodriguez-Torres, A. Osuna. Comparative sensitivity of six serological tests and diagnostic value of ELISA using purified antigen in hydatidosis.- J. Clin. Lab. Anal., 15, 2001, No 1, 14-18.

23. Singh, B. B., J. K. Sharma, S. Ghatak, R. Sharma, M. S. Bal, A. Tuli, et al. Molecular epidemiology of Echinococcosis from food producing animals in north India.- Vet. Parasitol., 186, 2012, No 3-4, 503-506.

24. Suwan, Z. Sonographic findings in hydatid disease of the liver: comparison with other imaging methods.- Ann. Trop. Med. Parasitol., 89, 1995, No 3, 261-269.

25. Tarnovetchi, C., G. S. Aprodu, M. Oancea. Diagnosis and treatment of abdominal hydatid cysts in children. A multicentric study.- Rev. Med. Chir. Soc. Med. Nat. Iasi, 114, 2010, No 4, 1087-1091.

26. Todorov, T., V. Boeva. Echinococcosis in children and adolescents in Bulgaria: a comparative study.Ann. Trop. Med. Parasitol., 94, 2000, No 2, 135-144.

27. Wen, H., T. Aji, Y. M. Shao. Diagnosis and management against the complications of human cystic echinococcosis.- Front. Med. China, 4, 2010, No 4, 394-398. 